\title{
Joint Structure Preserving Embedding Model and Its Application for Process Monitoring
}

\author{
Yuanjian $\mathrm{Fu}^{1 *}$, Chaomin $\mathrm{Luo}^{2}$
}

${ }^{1}$ College of Information Science and Engineering,

Northeastern University, Shenyang 110819, Liaoning,

\section{China}

2 Department of Electrical and Computer Engineering, Mississippi State University, Mississippi State, MS 39762, USA

*Corresponding author: huazi90927@126.com 
Appendix A. The deriving process

For the completeness of the paper, the deriving process for formula (15) is briefly summarized as follows:

$\left\|\tau\left(\mathbf{D}_{N}\right)-\tau\left(\mathbf{D}_{E}\right)\right\|_{L_{2}}^{2}$

$=\operatorname{tr}\left(\left(\tau\left(\mathbf{D}_{N}\right)-\tau\left(\mathbf{D}_{E}\right)\right)\left(\tau\left(\mathbf{D}_{N}\right)-\tau\left(\mathbf{D}_{E}\right)\right)^{T}\right)$

$=\operatorname{tr}\left(\left(\tau\left(\mathbf{D}_{N}\right) \tau\left(\mathbf{D}_{N}\right)^{T}-\tau\left(\mathbf{D}_{E}\right) \tau\left(\mathbf{D}_{N}\right)^{T}-\tau\left(\mathbf{D}_{N}\right) \tau\left(\mathbf{D}_{E}\right)^{T}+\tau\left(\mathbf{D}_{E}\right) \tau\left(\mathbf{D}_{E}\right)^{T}\right)\right.$ where $\tau\left(\mathbf{D}_{E}\right)=\mathbf{y}^{T} \mathbf{y}=\mathbf{X}^{T} \mathbf{a a}^{T} \mathbf{X}$, and the $=\operatorname{tr}\left(\left(\tau\left(\mathbf{D}_{N}\right) \tau\left(\mathbf{D}_{N}\right)^{T}-\mathbf{X}^{T} \mathbf{a a}^{T} \mathbf{X} \tau\left(\mathbf{D}_{N}\right)^{T}-\right.\right.$

$\left.\tau\left(\mathbf{D}_{N}\right) \mathbf{X}^{T} \mathbf{a a}^{T} \mathbf{X}+\mathbf{X}^{T} \mathbf{a a}^{T} \mathbf{X} \mathbf{X}^{T} \mathbf{a a}^{T} \mathbf{X}\right)$

$=\operatorname{tr}\left(\left(\tau\left(\mathbf{D}_{N}\right) \tau\left(\mathbf{D}_{N}\right)^{T}\right)-2 \operatorname{tr}\left(\mathbf{a}^{T} \mathbf{X} \tau\left(\mathbf{D}_{N}\right) \mathbf{X}^{T} \mathbf{a}\right)+1\right.$

magnitude of $\mathbf{a}$ is of no real significance because it only scales $\mathbf{y}$, so a constraint is imposed on $\mathbf{y}$,

$\mathbf{a}^{T} \mathbf{X} \mathbf{X}^{T} \mathbf{a}=1, \operatorname{tr}\left(\mathbf{X}^{T} \mathbf{a}^{T} \mathbf{X} \mathbf{X}^{T} \mathbf{a a}^{T} \mathbf{X}\right)=\operatorname{tr}\left(\mathbf{a}^{T} \mathbf{X} \mathbf{X}^{T} \mathbf{a a}^{T} \mathbf{X} \mathbf{X}^{T} \mathbf{a}\right)$.

Therefore, the minimization problem can be shown as:

$$
\max \mathbf{a}^{T} \mathbf{X} \tau\left(\mathbf{D}_{N}\right) \mathbf{X}^{T} \mathbf{a}
$$

where $\mathbf{D}_{N}$ is the geodesic distance matrix. 